\title{
Distinguishing adolescents who think about self-harm from those who engage in self-harm
}

Rory C. O'Connor, Susan Rasmussen and Keith Hawton

\section{Background}

Adolescent self-harm is a major public health concern, yet little is known about the factors that distinguish adolescents who think about self-harm but do not act on these thoughts from those who act on such thoughts.
Aims
Within a new theoretical model, the integrated motivational- volitional model, we investigated factors associated with adolescents having thoughts of self-harm (ideators) $v$. those associated with self-harm enaction (enactors).

\section{Method}
Observational study of school pupils employing an anonymous self-report survey to compare three groups of adolescents: self-harm enactors $(n=628) v$. self-harm ideators $(n=675) v$. those without any self-harm history $(n=4219)$.

\section{Results}

Enactors differed from ideators on all of the volitional factors. Relative to ideators, enactors were more likely to have a family member/close friend who had self-harmed, more likely to think that their peers engaged in self-harm and they were more impulsive than the ideators. Enactors also reported more life stress than ideators. Conversely, the two self-harm groups did not differ on any of the variables associated with the development of self-harm thoughts.

\section{Conclusions}

As more adolescents think about self-harm than engage in it, a better understanding of the factors that govern behavioural enaction is crucial in the effective assessment of the risk of self-harm.

\section{Declaration of interest}

None.
Self-harm (self-poisoning or self-injury) is a major public health concern, particularly in adolescents. ${ }^{1}$ It is one of the strongest predictors of completed suicide, ${ }^{2-4}$ with adolescents who self-harm four times more likely to die by suicide than those who have not self-harmed. ${ }^{5}$ Although considerable research has highlighted the characteristics of adolescents who self-harm, ${ }^{1,6-11}$ our understanding of many aspects of self-harm in young people is fragmented. Many more adolescents have thoughts of self-harm (ideators) than go on to engage in this behaviour (enactors), yet we know little about the factors that differentiate these two groups of adolescents. ${ }^{1,11}$ This is an important issue because of its relevance to the assessment of risk of self-harm.

The integrated motivational-volitional (IMV) model $^{12}$ is a new tripartite framework (including biological, psychological and social factors) that provides a theoretical basis for factors associated with the development of suicidal ideation and the translation of these thoughts into suicidal behaviour (Fig. 1). It integrates predominant factors from existing models including Williams' arrested flight model, ${ }^{13}$ with the diathesis-stress hypothesis $^{14}$ and a dominant model of health behaviour. ${ }^{15}$ Crucially, the conceptualisation of this model is that self-harm is not simply a by-product of clinical disorder ${ }^{12}$ but it is a behaviour that develops through three phases: the pre-motivational phase (consisting of background factors and triggering events that set the biosocial context), the motivational phase (consisting of factors associated with the formation of suicidal thoughts and intention to end one's life) and the volitional phase (consisting of factors associated with the translation of thought into action).

In brief, the IMV model posits that stressful life events that are appraised as being defeating or humiliating and that lead to feelings of entrapment increase the risk of suicidal ideation. Whether suicidal ideation emerges from entrapment, however, is determined by a range of factors, labelled motivational moderators, which include low levels of social support, few positive thoughts for the future and dysfunctional goal regulation. ${ }^{16-18}$ The psychological processes that underpin the transitions from defeat through to suicidal ideation are outlined in the motivational phase of the IMV model (Fig. 1). Finally, the volitional moderators are a distinct group of factors that increase the risk that an individual will act on their suicidal thoughts (i.e. engage in suicidal behaviour), the latter include impulsivity, access to the means of suicide and exposure to others who have engaged in suicidal behaviour. Although it was developed with suicidality in mind, the IMV model is applicable to understanding non-fatal self-harm irrespective of motive.

A key premise of the model is that the factors and processes underpinning the development of thoughts of self-harm are different from those associated with engaging in self-harm/suicidal behaviour. ${ }^{12}$ For example, brooding rumination (passively focusing on the reasons for personal distress), a motivational phase variable, may increase thoughts of self-harm as it leads to repetitious focus on negative aspects of life, thus contributing to a vicious cycle of despair. It is not associated, however, with behavioural enaction per se. Conversely, exposure to others who self-harm or beliefs about what individuals think others do (descriptive norms), both volitional phase variables, may be associated with behavioural enaction as they may provide a behavioural model that can bridge between thoughts and behaviour (descriptive norms are distinct from subjective norms (motivational phase variable), which are concerned with the social pressure to act in a particular way). Negative life events, despite being situated within the premotivational phase, are associated with behavioural enaction as well as ideation, because within a diathesis-stress framework, they act as triggering events to self-harm and as their occurrence increases so does the likelihood of behavioural enaction. Although the IMV model does not model developmental changes explicitly, the impact of puberty and brain maturation would fit within the pre-motivational phase, as the former provides the biosocial context for self-harm or suicide.

We have investigated a range of established risk factors for selfharm $^{1,11,19-22}$ to assess their relevance to the three phases of the IMV model ${ }^{12}$ in a sample of adolescents with and without a 


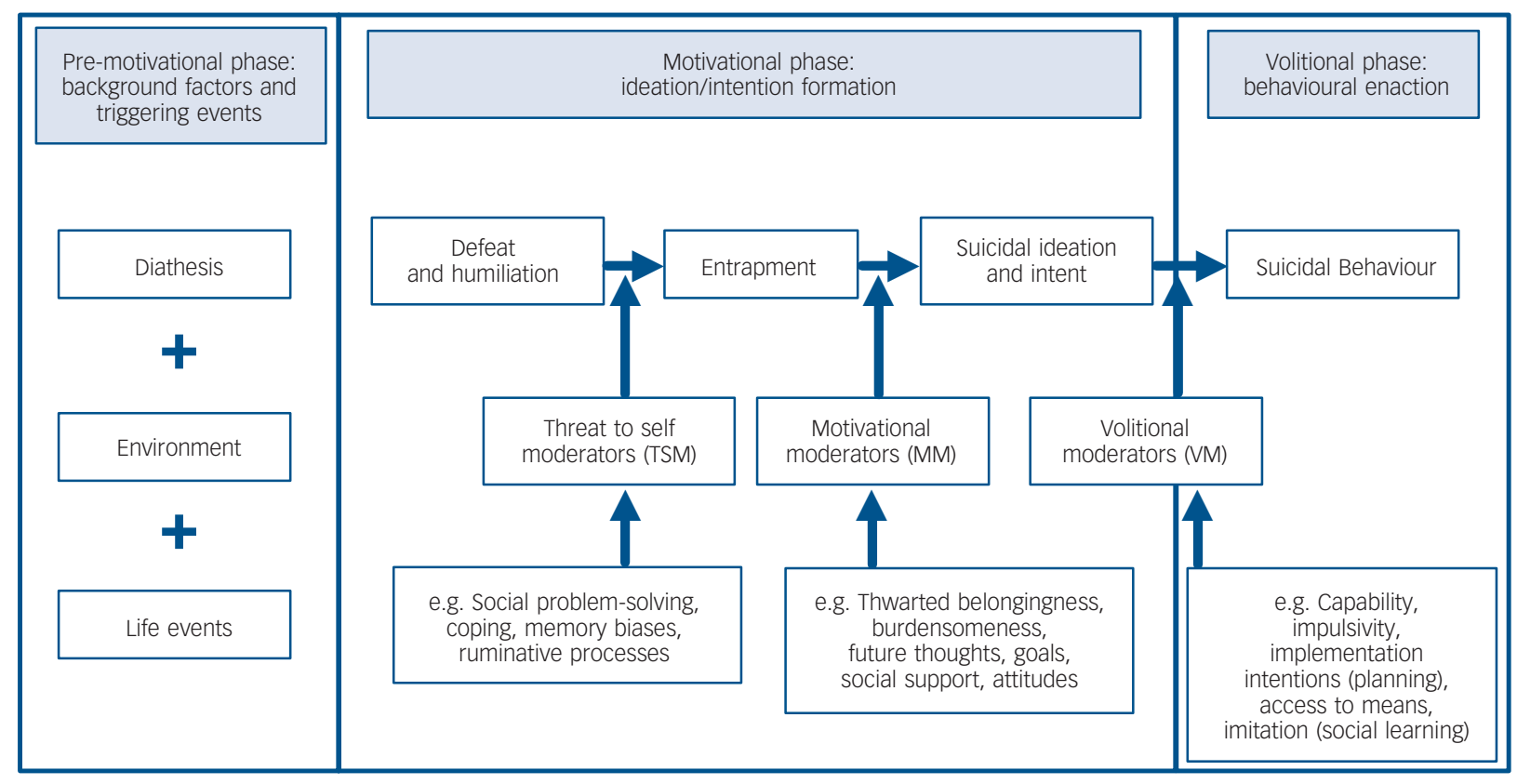

Fig. 1 Integrated motivational-volitional model of self-harm/suicidal behaviour. ${ }^{12}$

history of self-harm ideation/acts. Specifically, based on the IMV model we have tested the hypotheses that (a) ideators and enactors would differ significantly from controls (without thoughts/acts of self-harm) on the pre-motivational phase personality measures and motivational phase measures (namely, on socially prescribed perfectionism, self-esteem, brooding rumination, optimism), but there would be no differences between ideators and enactors on these measures; and (b) ideators and enactors would differ from controls on the volitional phase measures (namely, on descriptive norms, having family members and friends who have self-harmed in the past, impulsivity) and the experience of negative life events, but ideators would also differ from enactors on these measures.

\section{Method}

\section{Participants}

A total of 5604 school pupils (2776 female, 2821 male, 7 participants did not indicate their gender) were recruited from 41 secondary schools in Scotland (where 13/45 schools agreed to participate) and Northern Ireland (where 28/70 schools agreed to participate) as part of the Scotland and Northern Ireland Lifestyle and Coping Surveys. ${ }^{11,23,24}$ All secondary schools in Glasgow (west Scotland ) and Stirling (central Scotland) $(n=45)$ and a random sample of 70 of all the secondary schools in Northern Ireland were invited to participate. All pupils were in secondary years S4/Year 11 and S5/Year 12 and in classes in which $90 \%$ of the young people were aged $15-16$ years. This sample was broadly representative of the target populations in terms of school type, ethnic minorities, educational attainment and socioeconomic deprivation. The total sample represented approximately $80 \%$ of those eligible to participate. Timetable constraints and absenteeism were the main reasons for nonparticipation. Ethical approval was obtained from the Psychology Department's ethics committee at the University of Stirling. Parents were informed of the project by letter and asked to notify the school if they did not want their child to participate. Two or three weeks before data collection, the nature of participation was explained in detail to the teachers. On the day of participation, pupils were given the choice of opting out and not participating.
Respondents completed a modified version of the Child and Adolescent Self-harm in Europe (CASE) study questionnaire. ${ }^{1,25}$ This is an anonymous self-report questionnaire that takes approximately $30 \mathrm{~min}$ to complete. The original survey was developed in collaboration with experts in school-based studies and underwent extensive piloting in schools and an adolescent psychiatric unit. We only report on those measures pertinent to the present study here (see O'Connor et al for full details of procedure and measures). ${ }^{11,23}$

\section{Measures}

Self-harm acts were recorded if a respondent answered 'yes' (labelled enactor) to the following question: 'Have you ever deliberately taken an overdose (e.g. of pills or other medication) or tried to harm yourself in some other way (such as cut yourself)?. Self-harm thoughts were recorded if a respondent answered 'yes' (labelled ideator) to the following question: 'Have you ever seriously thought about taking an overdose or trying to harm yourself but not actually done so?' and 'no' to the selfharm act question. The ideator group, therefore, is only comprised of adolescents who had seriously thought about self-harm and have never acted on these thoughts. Mood (depression and anxiety) was assessed via the 14-item Hospital Anxiety and Depression Scale (HADS), ${ }^{26}$ which is validated for adolescents. ${ }^{27}$ Socially prescribed perfectionism assesses the degree of belief that others hold unrealistically high expectations of one's behaviour and that they would only be satisfied with these standards. It is assessed via the modified 7-item subscale of the Child and Adolescent Perfectionism Scale (CAPS). ${ }^{28}$

Brooding rumination assesses the extent to which individuals passively focus on the reasons for their distress. In addition, optimism and self-esteem were assessed via the revised Life Orientation Scale (LOT-R) ${ }^{29}$ and a modified version of the SelfConcept Scale ${ }^{30,31}$ respectively. Four variables that tap the volitional phase were also included: self-harm by friends, selfharm by family, descriptive norms and impulsivity. Respondents were asked the following questions about self-harm by close friends and family: 'Has anyone among your close friends [your family] attempted suicide or deliberately harmed themselves?' 
Four items assessed descriptive norms, ${ }^{32}$ defined as the beliefs about self-harm and the self-harm behaviour of peers and friends. Two items ('I do things on the spur of the moment' and 'I do things impulsively') from the Plutchik Impulsivity Scale assessed impulsivity. ${ }^{33}$ Negative life stress is a composite of 18 potentially stressful life events that are relevant to adolescent school children. ${ }^{24}$ The life events measure was developed following extensive piloting in schools and an adolescent psychiatric unit. ${ }^{31}$ Examples of events include 'Have you been bullied at school?' and 'Have you been seriously physically abused?' A higher score indicates the experience of more life events.

\section{Statistical analyses}

To test the two overarching hypotheses, a series of univariate multinomial logistic regression analyses were conducted in which we determined whether each pre-motivational, motivational or volitional phase variable differentiated between the three groups (ideators $v$. enactors $v$. controls). Given established gender differences, ${ }^{3}$ gender was controlled for in all analyses. To ensure that variations in mood and age did not account for the hypothesised effects, we also controlled for levels of depression and anxiety in all analyses. To control for the number of comparisons we employed Holm's sequential Bonferroni correction method. Finally, to adjust for potential clustering effects, we employed the Huber-White sandwich estimator method using logistic regression within the complex samples procedure in PASW (version 18) on Windows, specifying school as the clustering variable in all analyses. The analyses in Tables 1 and 2 are looking at changes in single points of continuous measures and are predicated on reasonably normally distributed responses.

\section{Results}

Of the overall sample of 5604 school pupils, 675 (12.2\%) reported thoughts of self-harm without acting upon them (ideators), 628 (11.4\%) reported acts of self-harm (enactors) and 4219 (76.4\%) reported no history of thoughts or acts (controls; 82 respondents did not answer either or both of the self-harm questions). Significantly more girls than boys were ideators ( $64 \%$ female, odds ratio $(\mathrm{OR})=2.09,95 \%$ CI $1.56-2.79, P=0.0001)$ and enactors (75\% female, OR $=3.73,95 \%$ CI $2.78-5.00, P=0.0001)$.

Both self-harm groups (ideators and enactors) differed significantly from the control group on socially prescribed perfectionism, brooding, self-esteem and optimism (i.e. the premotivational (background variables) and motivational phase variables) in the predicted directions (Table 1). In addition, ideators did not differ significantly from the enactors on any of these variables. The ideators and the enactors differed significantly from the control group and each other in the predicted directions on the volitional phase variables (self-harm by family, self-harm by friends, descriptive norms and impulsivity) and negative life stress (Table 2). The means and confidence intervals for each variable by self-harm status are presented in Table 3. Finally, when we entered those variables that significantly distinguished between ideators and enactors at the univariate level into a multivariate multinomial logistic regression to determine their relative contributions, being female $(\mathrm{OR}=1.51,95 \%$ CI 1.10-2.08, $P=0.014)$, having a family member $(\mathrm{OR}=2.60,95 \%$ CI 1.79-3.77, $P=0.0001)$ or close friend $(\mathrm{OR}=1.80,95 \%$ CI $1.28-2.52$, $P=0.001)$ who had self-harmed and negative life stress ( $\mathrm{OR}=1.05,95 \%$ CI $1.01-1.08, P=0.021)$ emerged as significant distinguishing factors. Depression $(\mathrm{OR}=1.04,95 \%$ CI $1.00-1.08$, $P=0.063)$ approached significance in the final model.

\section{Discussion}

\section{Main findings}

We found evidence to support both of our hypotheses. First, adolescents who were ideators or enactors differed significantly from the controls on the pre-motivational phase personality variables and motivational phase variables (i.e. socially prescribed perfectionism, self-esteem, brooding rumination and optimism). In addition, the ideators and enactors did not differ from each other on any of these measures. Second, it was the volitional phase variables (i.e. self-harm by family, self-harm by friends, descriptive norms and impulsivity) and the experience of negative life stress that distinguished the ideators from the enactors. Relative to ideators, those adolescents who acted on their thoughts of selfharm were significantly more likely to have a family member and/or close friend who had self-harmed, to believe that their

Table 1 Univariate multinomial logistic regression analyses (adjusting for clustering and controlling for age, mood and gender) of

\begin{tabular}{|c|c|c|c|c|}
\hline Pre-motivational and motivational phase variables & Self-harm status & OR & $95 \% \mathrm{Cl}$ & $P$ \\
\hline \multicolumn{5}{|l|}{ Socially prescribed perfectionism (scale range: $7-35)^{b, d}$} \\
\hline No self-harm behaviour or self-harm ideation & v. self-harm behaviour & 1.06 & $1.04-1.08$ & 0.0001 \\
\hline No self-harm behaviour or self-harm ideation & v. self-harm ideation & 1.06 & $1.04-1.08$ & 0.0001 \\
\hline Self-harm thoughts & v. self-harm behaviour & 1.01 & $0.99-1.03$ & 0.622 \\
\hline \multicolumn{5}{|l|}{ Brooding (scale range: $0-15)^{b, c, d}$} \\
\hline No self-harm behaviour or self-harm ideation & v. self-harm behaviour & 1.18 & $1.15-1.22$ & 0.0001 \\
\hline No self-harm behaviour or self-harm ideation & v. self-harm ideation & 1.21 & $1.17-1.26$ & 0.0001 \\
\hline Self-harm thoughts & v. self-harm behaviour & 1.03 & $0.99-1.06$ & 0.137 \\
\hline \multicolumn{5}{|l|}{ Self-esteem (scale range: $0-24)^{b, d}$} \\
\hline No self-harm behaviour or self-harm ideation & v. self-harm behaviour & 0.86 & $0.84-0.89$ & 0.0001 \\
\hline No self-harm behaviour or self-harm ideation & v. self-harm ideation & 0.87 & $0.84-0.89$ & 0.0001 \\
\hline Self-harm thoughts & v. self-harm behaviour & 1.00 & $0.96-1.03$ & 0.744 \\
\hline \multicolumn{5}{|l|}{ Optimism (scale range: $6-30)^{\mathrm{b}, \mathrm{d}}$} \\
\hline No self-harm behaviour or self-harm ideation & v. self-harm behaviour & 0.92 & $0.90-0.94$ & 0.0001 \\
\hline No self-harm behaviour or self-harm ideation & v. self-harm ideation & 0.92 & $0.90-0.94$ & 0.0001 \\
\hline Self-harm thoughts & v. self-harm behaviour & 1.00 & $0.97-1.02$ & 0.858 \\
\hline
\end{tabular}




\begin{tabular}{|c|c|c|c|c|}
\hline Volitional phase variables and negative life events & Self-harm status & OR & $95 \% \mathrm{Cl}$ & $P$ \\
\hline \multicolumn{5}{|l|}{ Self-harm in the family ${ }^{\mathrm{b}}$} \\
\hline No self-harm behaviour or self-harm ideation & v. self-harm behaviour & 7.27 & $5.84-9.05$ & 0.0001 \\
\hline No self-harm behaviour or self-harm ideation & v. self-harm ideation & 2.45 & $1.88-3.20$ & 0.0001 \\
\hline Self-harm thoughts & v. self-harm behaviour & 2.97 & $2.14-4.11$ & 0.0001 \\
\hline \multicolumn{5}{|l|}{ Self-harm by friends ${ }^{c}$} \\
\hline No self-harm behaviour or self-harm ideation & v. self-harm behaviour & 6.43 & $5.05-8.20$ & 0.0001 \\
\hline No self-harm behaviour or self-harm ideation & v. self-harm ideation & 2.76 & $2.23-3.41$ & 0.0001 \\
\hline Self-harm thoughts & v. self-harm behaviour & 2.33 & $1.75-3.12$ & 0.0001 \\
\hline \multicolumn{5}{|l|}{ Descriptive norms (scale range: $0-28)^{d}$} \\
\hline No self-harm behaviour or self-harm ideation & v. self-harm behaviour & 1.17 & $1.13-1.22$ & 0.0001 \\
\hline No self-harm behaviour or self-harm ideation & v. self-harm ideation & 1.11 & $1.06-1.16$ & 0.0001 \\
\hline Self-harm thoughts & v. self-harm behaviour & 1.06 & $1.03-1.09$ & 0.0001 \\
\hline \multicolumn{5}{|l|}{ Impulsivity (scale range: $0-6)^{d}$} \\
\hline No self-harm behaviour or self-harm ideation & v. self-harm behaviour & 1.29 & $1.20-1.38$ & 0.0001 \\
\hline No self-harm behaviour or self-harm ideation & v. self-harm ideation & 1.17 & $1.09-1.25$ & 0.0001 \\
\hline Self-harm thoughts & v. self-harm behaviour & 1.10 & $1.02-1.20$ & 0.017 \\
\hline \multicolumn{5}{|l|}{ Negative life stress (scale range: $0-18)^{d}$} \\
\hline No self-harm behaviour or self-harm ideation & v. self-harm behaviour & 1.49 & $1.43-1.57$ & 0.0001 \\
\hline No self-harm behaviour or self-harm ideation & v. self-harm ideation & 1.33 & $1.27-1.38$ & 0.0001 \\
\hline Self-harm thoughts & v. self-harm behaviour & 1.13 & $1.09-1.17$ & 0.0001 \\
\hline \multicolumn{5}{|c|}{$\begin{array}{l}\text { a. Each of the pair-wise comparisons that is significant remains significant after applying Holm's sequential Bonferroni correction method. } \\
\text { b. Exposure to family self-harm }(n) \text { is } 309 / 4201,142 / 673,295 / 626 \text { in the no self-harm history, ideator and enactor groups, respectively. } \\
\text { C. Exposure to friends' self-harm }(n) \text { is } 687 / 4207,280 / 673,404 / 623 \text { in the no self-harm history, ideator and enactor groups, respectively. } \\
\text { d. As these are dimensional/continuous measures, the odds ratio represents each one point increase in score. }\end{array}$} \\
\hline
\end{tabular}

friends/peers engaged in self-harm (descriptive norms) and they were significantly more impulsive. Our findings are not explained by mood or age variations across the groups nor can they be attributed to clustering within schools. Moreover, in the final multivariate analyses, we included all univariate predictors as explanatory variables (including age, gender, anxiety and depression), and neither index of mood entered the final model to distinguish enactors from ideators (although there was a non-significant trend for depression).

We have extended the findings of previous studies of self-harm in adolescents considerably by providing evidence for a priori hypotheses derived from a theoretical model that makes specific predictions about the types of factors that are associated with behavioural enaction of self-harm. ${ }^{1,9,11,34}$ This is a significant advance as it moves beyond describing characteristics of self-harm phenomena to formulating inferential, theory-driven hypotheses about those factors that are associated with the development of self-harm thoughts $v$. those factors that facilitate the behavioural enaction of those thoughts. This is also the first study to demonstrate that motivational factors do not distinguish ideators from enactors, rather that volitional factors and the extent of life stress seem to govern behavioural enaction, determining when thoughts of self-harm are translated into acts of self-harm. Of the volitional factors, having family or friends who have selfharmed was statistically the most important. This is in keeping with the notion that contagion is an important influence on self-harm in adolescents. ${ }^{35}$ Although the rate of ideation is often reported to be higher than enaction in studies of this kind, ${ }^{14}$ the lower rate of ideation in the present study reflects the fact that our ideator group is comprised only of those adolescents who have reported serious ideation and never acted on such thoughts, i.e. they are an ideator-only group.

\section{Strengths and limitations}

Key strengths of the study include it being based on a theoretical model of self-harm/suicidal behaviour and it comprises a large representative community sample of adolescents. However, as the

Table 3 Means (and 95\% confidence intervals adjusting for clustering) by self-harm status controlling for age, mood and gender

\begin{tabular}{|c|c|c|c|}
\hline \multirow[b]{2}{*}{ Variable } & \multicolumn{3}{|c|}{ Mean $(95 \% \mathrm{Cl})$} \\
\hline & $\begin{array}{l}\text { No self-harm behaviour } \\
\text { or self-harm ideation }\end{array}$ & Self-harm thoughts & Self-harm behaviour \\
\hline \multicolumn{4}{|c|}{ Pre-motivational and motivational phase variables } \\
\hline Socially prescribed perfectionism & $18.50(18.10-18.90)$ & $21.61(20.94-22.28)$ & $22.25(21.71-22.78)$ \\
\hline Self-esteem & $16.07(15.97-16.18)$ & $13.10(12.82-13.38)$ & $12.31(12.06-12.55)$ \\
\hline Brooding rumination & $4.91(4.71-5.11)$ & $7.94(7.65-8.22)$ & $8.14(7.82-8.47)$ \\
\hline Optimism & $19.71(19.54-19.89)$ & $17.32(17.01-17.63)$ & $16.84(16.56-17.12)$ \\
\hline \multicolumn{4}{|c|}{ Voltional phase variables and negative life stress } \\
\hline Self-harm by family ${ }^{a}$ & $0.07(0.06-0.08)$ & $0.21(0.16-0.26)$ & $0.47(0.42-0.52)$ \\
\hline Self-harm by friends ${ }^{a}$ & $0.16(0.14-0.19)$ & $0.42(0.36-0.47)$ & $0.65(0.60-0.70)$ \\
\hline Descriptive norms & $4.55(4.48-4.63)$ & $5.29(5.09-5.49)$ & $6.18(5.83-6.54)$ \\
\hline Impulsivity & $2.87(2.81-2.93)$ & $3.22(3.06-3.38)$ & $3.44(3.26-3.62)$ \\
\hline Negative life stress & $4.04(3.88-4.20)$ & $6.66(6.41-6.91)$ & $7.92(7.72-8.13)$ \\
\hline
\end{tabular}


present study has demonstrated, only an association between volitional phase factors and retrospective thoughts and behaviours, a large-scale prospective study is required to determine the extent to which volitional moderators and negative life stress actually predict those individuals with thoughts of self-harm who go on to engage in self-harm. Also, the volitional factors included here are not exhaustive; future research is required before we can generate a more definitive list of volitional factors that govern behavioural enaction. We also relied on self-reported incidence of thoughts of self-harm and actual self-harm. Despite the large sample of adolescents included in the study, the vast majority of those who reported/thought about self-harm were female thereby precluding reliable group analyses by gender in the present study.

\section{Implications}

This study provides support for the tripartite IMV model of selfharm/suicidal behaviour. It also helps establish self-harm as a 'health' behaviour, not just a sign of clinical disorder. Consequently, future research should examine the extent to which self-harm is amenable to established behaviour change techniques ${ }^{36}$ as well as traditional therapeutic approaches. It also reinforces the view that we need to move beyond just psychiatric diagnosis to better understand self-destructive behaviours. ${ }^{12,37}$ It is also worth highlighting that the large sample size cannot account for the statistically significant differences between ideators and enactors on the univariate volitional phase variables. If these differences were statistical artefacts, one would expect to find similar differences among the pre-motivational phase personality and motivational phase variables. No such differences were evident. The implications for clinical practice and prevention are also considerable.

First, the present findings suggest that it may be important in therapy to use psychosocial interventions that differentially address the pre-motivational/motivational and the volitional phases of self-harm. For example, a cognitive-behavioural intervention could address some of the underlying processes that contribute to the development of thoughts of self-harm. ${ }^{38}$ These may include tackling perfectionistic beliefs or brooding rumination. In addition, a behaviour change-based intervention may be used to address the effect of exposure to self-harm on behavioural enaction. ${ }^{36,39}$ From a public health perspective one might consider interventions to try to modify adolescent norms associated with self-harm.

Second, these findings also provide guidance on the types of questions and issues that clinicians may wish to address if a young person discloses having thoughts of self-harm. Asking about exposure to self-harm by others could be beneficial, as could a closer inspection of the individual's propensity to be impulsive.

Given the apparent role of so-called exposure or social learning variables (i.e. self-harm of others) in the aetiology of self-harm enaction, future research should investigate potential mechanisms to explain how they bridge the self-harm thoughtsbehaviour gap. In another component of the present study, we obtained some evidence for social learning and legitimisation of self-harm explanations (such as an adolescent saying if it is okay for my mother to self-harm when she is under pressure, it is okay for me to do so as well'). ${ }^{22}$ Using data from the CASE study in England, Hargus et $a l^{34}$ found that adolescents who explained their self-harm in terms of being suicidal were more likely to have family members who had died by suicide than those who explained their self-harm in other ways, who were more likely to have friends who had self-harmed. Thus, the context and nature of the exposure to others who have self-harmed may be important. We also need to determine the role of such factors in repeat self-harm $v$. single-episode self-harm as well as among those expressing suicidal intent $v$. those who do not.

Rory C. O'Connor, PhD, Suicidal Behaviour Research Group, School of Natural Sciences, University of Stirling, Stirling; Susan Rasmussen, PhD, School of Psychological Sciences and Health, University of Strathclyde, Glasgow; Keith Hawton, DSc, Centre for Suicide Research, Department of Psychiatry, University of Oxford, Warneford Hospital, Oxford, UK

Correspondence: Rory C. O'Connor, Suicidal Behaviour Research Group, School of Natural Sciences, University of Stirling, Stirling FK9 4LA. Email: ro2@stir.ac.uk

First received 10 Jun 2011, final revision 17 Nov 2011, accepted 19 Dec 2011

\section{Funding}

The Northern Ireland Lifestyle and Coping Survey was funded by the Department of Health, Social Services and Public Safety (NI) and supported by the Department of Education (NI).

\section{Acknowledgements}

Thanks to staff and pupils at the schools in Scotland and Northern Ireland for their participation. Thanks also to Michelle Scott who coordinated the data collection in Northern Ireland and Dr Jeremy Miles for statistical advice. K.H. is a National Institute for Health Research Senior Investigator.

\section{References}

1 Hawton K, Rodham K, Evans E, Weatherall R. Deliberate self-harm in adolescents: self-report survey in schools in England. BMJ 2002; 325: 1207-11.

2 Hawton $K$, Harriss $L$, Hall S, Simkin S, Bale E, Bond A. A time of change in patient characteristics. Psychol Med 2003; 33: 987-96.

3 Hawton K, van Heeringen K. Suicide. Lancet 2009; 373: 1372-81.

4 Owens D, Horrocks J, House A. Fatal and non-fatal repetition of self-harm. Systematic review. Br J Psychiatry 2002; 181: 193-9.

5 Goldacre M, Hawton K. Repetition of self-poisoning and subsequent death in adolescents who take overdoses. Br J Psychiatry 1985; 146: 395-8.

6 Cloutier P, Martin J, Kennedy A, Nixon MK, Muehlenkamp JJ. Characteristics and co-occurrence of adolescent non-suicidal self-injury and suicidal behaviours in pediatric emergency crisis services. $J$ Youth Adolesc 2010; 39: 259-69.

7 Evans E, Hawton K, Rodham, K. Factors associated with suicidal phenomena in adolescents: a systematic review of population-based studies. Clin Psychol Rev 2004; 24: 957-79.

8 Gould MS, King R, Greenwald S, Fisher P, Schwab-Stone M, Kramer R, et al. Psychopathology associated with suicidal ideation and attempts among children and adolescents. J Am Acad Child Adolesc Psychiatry 1998; 37 915-23.

9 McMahon EM, Reulbach U, Corcoran P, Keeley HS, Perry IJ, Arensman E. Bullying victimisation, self-harm and associated factors in Irish adolescent boys. Soc Sci Med 2010; 71: 1300-7.

10 O'Connor RC, Rasmussen S, Hawton K. Predicting deliberate self-harm in adolescents: a six month prospective study. Suicide Life Threat Behav 2009; 39: $364-75$.

11 O'Connor RC, Rasmussen S, Miles J, Hawton K. Self-harm in adolescents: self-report survey in schools in Scotland. Br J Psychiatry 2009; 194: 68-72.

12 O'Connor RC. Towards an integrated motivational-volitional model of suicidal behaviour. In International Handbook of Suicide Prevention: Research, Policy \& Practice (eds RC O'Connor, S Platt, J Gordon): 181-98. John Wiley \& Sons, 2011

13 Williams JMG. The Cry of Pain. Penguin, 2001.

14 Schotte DE, Clum GA. Problem solving skills in suicidal psychiatric patients. J Consult Clin Psychol 1987; 55: 49-54.

15 Ajzen I. The theory of planned behavior. Organ Behav Hum 1991; 50: 179-211.

16 Rasmussen S, Fraser L, Gotz M, MacHale S, Mackie R, Masterton G, McConacie $S$, et al. Elaborating the cry of pain model of suicidality: testing a psychological model in a sample of first-time and repeat self-harm patients. Br J Clin Psychol 2010; 49: 15-30.

17 O'Connor RC, Rasmussen S, Hawton K. Predicting depression, anxiety and self-harm in adolescents: the role of perfectionism and acute life stress. Behav Res Ther 2010; 48: 52-9. 
18 O'Connor RC, Fraser L. Whyte MC, MacHale S, Masterton G. Self-regulation of unattainable goals in suicide attempters: the relationship between goal disengagement, goal reengagement and suicidal ideation. Behav Res Ther 2009; 47: 164-9.

19 Portzky G, De Wilde EJ, Van Heeringen K. Deliberate self-harm in young people: differences in prevalence and risk factors between The Netherlands and Belgium. Eur Child Adolesc Psychiatry 2007; 17: 179-86.

20 Morey C, Corcoran P, Arensman E, Perry IJ. The prevalence of self-reported deliberate self harm in Irish adolescents. BMC Public Health 2008; 8: 79.

21 De Leo D, Heller ST. Who are the kids who self-harm? An Australian self-report survey. Med J Aust 2004; 181: 140-4.

22 O'Connor RC, Fraser L, Whyte MC, MacHale S, Masterton G. A comparison of specific positive future expectancies and global hopelessness as predictors of suicidal ideation in a prospective study of repeat self-harmers. J Affect Disord 2008; 110: 207-14.

23 O'Connor RC, Rasmussen S, Hawton K. Northern Ireland Lifestyle and Coping Survey. Final Report. Department of Health, Social Services and Public Safety 2010 (http://www.dhsspsni.gov.uk/ni-lifestyle-and-coping-survey-2010.pdf).

24 O'Connor RC, Rasmussen S, Hawton K. Predicting depression, anxiety and self-harm in adolescents: the role of perfectionism and acute life stress. Behav Res Ther 2010; 48: 52-9.

25 Madge N, Hewitt A, Hawton K, de Wilde EJ, Corcoran P, Fekete S, et al. Deliberate self-harm within an international community sample of young people: findings from the Child \& Adolescent Self-harm in Europe (CASE) study. J Child Psychol Psychiatry 2008; 49: 667-77.

26 Zigmond AS, Snaith RP. The hospital anxiety and depression scale. Acta Psychiatr Scand 1983; 67: 361-70.

27 White $D$, Leach R, Sims R, Atkinson M, Cottrell D. Validation of the Hospital Anxiety and Depression Scale for use with adolescents. Br J Psychiatry 1999; 175: $452-4$.
28 O'Connor RC, Dixon D, Rasmussen S. The structure and temporal stability of the child and adolescent perfectionism scale. Psychol Assess 2009; 21: 43743.

29 Scheier MF, Carver CS, Bridges MW. Distinguishing optimism from neuroticism (and trait anxiety, self-mastery and self-esteem): a re-evaluation of the Life Orientation Test. J Pers Soc Psychol 1994; 67: 1063-78.

30 Robson P. Development of a new self-report questionnaire to measure self-esteem. Psychol Med 1989; 19: 513-8.

31 Hawton K, Rodham K, Evans E. By Their Own Young Hand: Deliberate SelfHarm and Suicidal Ideas in Adolescents. Jessica Kingsley Publishers, 2006.

32 O'Connor RC, Armitage CJ, Gray L. The role of clinical and social cognitive variables in parasuicide. Br J Clin Psychol 2006; 45: 465-81.

33 Plutchick R, van Praag HM, Picard S, Conte HR, Korn M. Is there a relation between the seriousness of suicidal intent and the lethality of the suicide attempt? J Psychiatr Res 1989; 27: 71-9.

34 Hargus E, Hawton K, Rodham, K. Distinguishing between subgroups of adolescents who self-harm. Suicide Life Threat Behav 2009; 39: 518-37.

35 Hawton K, Harriss L, Rodham K. How adolescents who cut themselves differ from those who take overdoses. Eur Child Adolesc Psychiatry 2010; 19: 513-23.

36 Abraham C, Michie S. A taxonomy of behavior change techniques used in interventions. Health Psychol 2008; 27: 379-87.

37 Van Heeringen $\mathrm{K}$. Towards a psychobiological model of the suicidal process. In Understanding Suicidal Behaviour (ed K van Heeringen): 136-59. John Wiley \& Sons, 2001

38 Wenzel A, Brown GK, Beck AT. Cognitive Therapy for Suicidal Patients: Scientific and Clinical Applications. APA, 2009.

39 Dixon D, Johnston M. Health Behaviour Change Competency Framework: Competences to Deliver Interventions to Change Lifestyle Behaviours that Affect Health. Health Scotland, 2010 (http://www.healthscotland.com/ uploads/documents/14543-HBCC_framework1.pdf).

\section{0 words}

\section{Baby blues}

\section{Roch Cantwell}

Does common mean normal? If 50-80\% of women experience transient mood lability, weepiness and irritability in the early postpartum, can we call it illness? Baby blues has dropped off the radar a little in recent years, yet in the past 'this trivial disorder has aroused almost as much curiosity as puerperal psychosis' (Brockington). Research has brought hints but no clarity to associations with predictable hormonal or neurochemical changes, but a stubborn link to postpartum depression remains. Will interest wane further, or can preoccupation with the 'pinks', brief postpartum mood elevation, bring a new hue to the spectrum of peripartum disorders? 